Censure et productions culturelles postcoloniales

\title{
Nabile Farès et la censure: du silence des mots aux échos des images
}

Nabile Farès and Censorship: From Silenced Words to Visual Echoes

\section{Karine Chevalier}

\section{(2) OpenEdition}

\section{Journals}

Édition électronique

URL : http://journals.openedition.org/transtexts/1412

DOI : $10.4000 /$ transtexts. 1412

ISSN : 2105-2549

Éditeur

Gregory B. Lee

\section{Référence électronique}

Karine Chevalier, "Nabile Farès et la censure: du silence des mots aux échos des images », Transtext(e)s Transcultures 跨文本跨文化 [En ligne], 15 | 2020, mis en ligne le 14 décembre 2020, consulté le 27 janvier 2021. URL : http://journals.openedition.org/transtexts/1412 ; DOI : https:// doi.org/10.4000/transtexts. 1412

Ce document a été généré automatiquement le 27 janvier 2021.

(c) Tous droits réservés 


\title{
Nabile Farès et la censure: $\mathrm{du}$ silence des mots aux échos des images
}

Nabile Farès and Censorship: From Silenced Words to Visual Echoes

\author{
Karine Chevalier
}

1 Poète, anthropologue, sociologue, psychanalyste, professeur et chercheur, ces quelques qualificatifs ne permettent que partiellement de faire le portrait de Nabile Farès (1940-2016). Personne inclassable, personnalité sensible et écrivain incontournable, Farès a connu de nombreuses censures, comme le rappellent certains évènements autobiographiques dont ses récits témoignent à demi-mots. Qu'il s'agisse de la censure idéologique qui ne respecte ni la différence ni le multiple (telle qu'il la découvrira lors de son engagement dans l'A.L.N), de l'ombre pesante de son père absent (Abderrahmane Farès, acteur politique clef de la libération algérienne, arrêté et placé en résidence surveillée en 1964 par le premier président de l'Algérie indépendante), du massacre du 17 Octobre 1961 (gangrène historique encore non assumée par l'État français) ou encore du statut problématique de la langue et de la culture berbères, Farès n'a eu de cesse de révéler les refoulements mémoriels aussi bien individuels que collectifs. ${ }^{1}$ Se positionnant contre le parti unique et le monolinguisme, il veut comprendre les origines de cette intolérance et de la violence qui en découle, déflagrant les sociétés, les individus, les paysages. Il cherche l'origine de la monstruosité (du radicalisme au racisme) qui a imposé ces censures dans les guerres, les mythes, les contes, l'étymologie des mots. Son écriture a toujours voulu témoigner de ces déflagrations à partir de traces polyphoniques, de bribes de sens pour faire parler l'enfance, l'inconscient, le chaos, en quête de signes protecteurs, en cherchant à proposer un langage multiple contre les langages de l'Un au risque d'être une «surface gondolée présente où se dérobe le parler manifeste dans la manifestation de la parole, étourdissante dialectique de l'absence où celui qui parle est caché dans le limon des mots ». ${ }^{2}$ Comment ces censures historiques, idéologiques, linguistiques, politiques entraînent-elles une écriture volontairement opaque au niveau du signifiant et du 
signifié et comment cette écriture de l'(auto-)censure permet-elle de détourner ces censures?

2 Cette écriture de l'absent, qu'il n'aimait pas commenter (et encore moins traduire), lui aura valu une censure éditoriale. « Il a toujours considéré les accusations qui font de son œuvre un travail hermétique comme une politique de censure, voire comme une censure politique " selon Ali Chibani. ${ }^{3}$ C'est donc grâce à des éditeurs indépendants (Maspero, Achab), ses nouveaux manuscrits ayant été refusés par Le Seuil, qu'il pourra continuer son travail, jusqu'à élargir dans ses dernières œuvres sa parole à la bande dessinée. Ce support intermédial illustre les enjeux d'une nouvelle stratégie polyphonique et polygraphique afin d'aller en deçà et au-delà de la censure. Comment s'est effectué ce passage du silence des mots aux échos des images et quels sont les enjeux engendrés au niveau du pacte de lecture ? Nous décrirons ce passage du silence des mots comme (auto-)censure aux échos des images comme nouvelle poésie visuelle. Nous analyserons ensuite comment s'ancre/s'encre la trace des absents en imposant une lecture en palimpseste pour conclure à un nécessaire dialogue phénoménologique et éthique comme contre-pouvoir aux censures.

\section{(Auto-)censure des mots et poésie visuelle}

3 Comme nous l'avons analysé dans La Mémoire et l'Absent, l'écriture de Farès a été motivée dès l'origine par un questionnement sur la parole refoulée afin de révéler les non-dits, les mal-dits, les maux-dits. ${ }^{4} \mathrm{Si}$ les mots évoquent la thématique du refoulement et de la mémoire déflagrée, ils servent plus souvent à illustrer cette parole en crise, fragmentée, délirante. Un des exemples les plus significatifs se trouve dans Le Champ des oliviers avec le dialogue allégorique (voire parodique) de deux frères siamois qui désirent se séparer, l'un représentant le signifiant et l'autre le signifié. Leur séparation symbolise la crise du langage quand «se dérobe le parler manifeste dans la manifestation de la parole", illustrée par une suite illogique et illisible de signes typographiques (tirets, parenthèses, chiffres, points-virgules, symboles de pourcentage). ${ }^{5}$ Cette conception d'une écriture qui cache le sens sous cette surface de traits d'encre témoigne de la déflagration historique et psychique à travers un questionnement sémiologique. Par la déconstruction grammaticale, l'utilisation des signes de ponctuation, de l'italique, des majuscules, des pages blanches ou encore des dessins et des calligrammes, les récits de Farès ont toujours requis une lecture graphique de l'encre imprimée sur le papier. Alors que le signifié des mots se fait polysémique, parfois à la limite de la compréhension, et appelle à l'interprétation, l'écriture farèsienne replace le signifiant au cœur de la communication pour rappeler la censure et le renouvellement nécessaire du pacte de lecture.

Il se trouve toujours des gens pour dire.

Pour me dire. Qu'ils ne comprennent pas.

Non.

Qu'ils ne comprennent pas mon langage ou

La

Forme de mon langage. ${ }^{6}$

Cette (auto)-censure qui appelle à une lecture graphique ne va que s'intensifier au fil de son œuvre, jusqu'à ses dernières productions qui feront dominer l'image sur les mots. 
5 En conséquence de la censure éditoriale qui rend son travail moins accessible au grand public, Farès s'est tourné vers la bande dessinée, choix délibéré de développer l'importance du signifiant visuel. C'est grâce à sa rencontre avec Kamel Khélif, son alter-ego (comme lui Algérien immigré en France après la guerre), qu'il va explorer ce medium. Encore considérée par certains comme un art mineur qui ne permet pas de proposer une pensée complexe, malgré un regain d'intérêt populaire (voire académique), la bande dessinée peut servir de plateforme à l'expression d'une poésie visuelle. En effet, l'approche graphique de Khélif se distingue par son opacité et rappelle ainsi l'écriture de Farès par ce choix esthétique. C'est de nouveau grâce à l'existence d'éditeurs indépendants dits alternatifs (Rackham, Amok) que la poésie visuelle trouvera sa place dans le monde de la bande dessinée. ${ }^{7}$ Cette poétique graphique évoluera chez Farès et Khélif. Ils se sont progressivement détournés de la figuration pour privilégier l'opacité et ainsi une lecture exigeante. Dans leur première collaboration, Les Exilées, histoires, les images illustraient de manière très réaliste le récit qui se faisait plus descriptif qu'à l'habitude pour évoquer la pauvreté, l'exode rural, l'émigration, l'insécurité après l'indépendance en Algérie. ${ }^{8}$ La Petite Arabe qui aimait la chaise de Van Gogh est plus abstrait, jouant sur la contradiction entre des images qui évoquent la désolation, les décombres, à partir de blocs d'encre noire délavée et des traits fins qui cisaillent cet espace monochrome alors que le texte évoque le «bleu de la mer ", «les roses jaunes" du paysage et «ces petits bouts de riens, paroles, et, déchets » « sur lesquels [...la petite arabe] avait fini par porter, à partir de la fabrication maladroite de pinceaux, des taches de couleur, des couches de peinture, des formes " pour " survivre ». ${ }^{9}$ Les mots évoquent ainsi un espace graphique que le lecteur se devra d'imaginer face à des traces d'encre noire, manière efficace d'illustrer l'opposition entre un espace extérieur visible et sombre et un espace intérieur imaginaire et coloré. Alors que dans Les Exilées Khélif dépasse ses angoisses et ose enfin représenter le port d'Alger, dans La Petite Arabe, le dessin plus abstrait laisse également place à de plus en plus d'espaces blancs. Le texte se raréfie. Presque une dizaine de pages sont laissées blanches, soit un tiers du livre, offrant un écran imaginaire vide que le lecteur peut remplir en réaction aux quelques lignes de texte en bas de page. Nul n'est besoin de rappeler au lecteur familier de l'écriture farèsienne que la page blanche est utilisée par l'écrivain pour évoquer le silence paradoxal: celui d'un trou mémoriel, d'un effacement mais également d'une vision initiatique. Ainsi, leur collaboration a évolué de manière organique, passant d'une approche traditionnelle à une conception plus abstraite et poétique de la bande-dessinée pour aboutir à La Jeune Femme et la mort se situant entre ces deux tendances. ${ }^{10}$ Pour autant, l'équilibre entre mots et dessins ne semble pas évident au premier abord. Avec quarante-six planches, les pages comportent entre deux et trois vignettes, majoritairement rectangulaires, étirées horizontalement, privilégiant ainsi un angle large pour les paysages ou semi-large pour situer les personnages dans l'espace. Le texte imprimé en haut ou en bas des vignettes ne constitue qu'un dixième de chaque planche et la typographie utilisée rappelle le caractère manuscrit, offrant une tonalité plus subjective. Cette domination de l'image sur le texte entraîne un nouveau rapport entre le lecteur et le livre. Il s'agit notamment de mieux comprendre les enjeux d'une écriture qui doit faire place aux dessins de Khélif en se demandant si ces dessins ont pour fonction d'amplifier le sens des mots de Farès alors que paradoxalement le travail de cet écrivain a toujours été d'en révéler les silences. 
6 Avec une approche graphique aussi opaque que l'écriture farèsienne, il est certain que le lecteur se trouvera devant une œuvre exigeante. Le passage d'une parole déflagrée à une parole dessinée, déjà à l'œuvre dans le travail poétique de Farès, continuera avec Kéhlif, en quête d'un juste équilibre entre réalisme et abstraction, paroles et silences, afin de créer un nouveau langage. Cette collaboration aura été rendue possible par un renouveau de la bande dessinée, considérée comme une production artistique aux codes graphiques innovants et au message politique engagé, d'une parole en marge mais assumée par des maisons d'édition alternatives. Il s'agit notamment pour les éditions Amok de défendre « une création artistique qui allie vision personnelle et souci des réalités contemporaines, sociales, historiques ou culturelles", position qui correspond à l'approche farèsienne de la fonction de l'écriture. ${ }^{11}$ Comment le travail de Farès et Khélif permet-il de représenter la trace des absents de manière renouvelée, du silence des mots aux échos des images? Comment dénoncer et détourner la censure par une écriture qui se veut passage de la trace au palimpseste pour révéler la mémoire des absents (au sens large, ceux qu'on a censurés dans leur mutisme, leur folie, leur exil...) ? C'est à partir de l'exemple de leur dernière collaboration, La Jeune Femme et la mort, que nous tenterons d'y répondre.

\title{
La trace des absents entre encre et ancrage : une lecture en palimpseste.
}

7 Comme le confirme Khélif en résumant le contenu narratif de La Jeune Femme et la mort, Farès est toujours parti d'évènements réels singuliers que ce soit des faits divers ou des évènements dits historiques, pour mélanger la micro-histoire avec la macro-Histoire.

\begin{abstract}
Nabile Farès a écrit ce livre à partir d'un fait-divers survenu en Algérie pendant les années 90, du côté de Médéa. C'est l'histoire d'une femme qui a 40 ans, elle vit avec sa petite fille. Depuis sa terrasse, elle voit le matin une fumée qui monte dans le ciel, elle aperçoit la cendre qui retombe sur le village où elle se trouve. Un souvenir lui revient : elle était quelques jours auparavant le témoin de l'assassinat d'un jeune couple, à l'intérieur de l'école où elle avait fait toutes ses classes. Il s'agit aussi de l'école où sa petite fille vient étudier. Cette histoire n'est pas du tout linéaire, elle est entrecoupée par des tas d'interrogations, il y a d'autres épisodes et d'autres histoires : par exemple, l'histoire des tribus qui ont vécu dans ce territoire et qui se sont massacrées. ${ }^{12}$
\end{abstract}

8 Farès fait ainsi de l'Histoire avec de multiples histoires. C'est ce que nous avions proposé d'appeler l'écriture de "traces historiques" dans La Mémoire et l'Absent, puisqu'elles ne sont pas historiquement explicitées mais plutôt appréhendées dans une constellation plus large d'évènements, de narrations, de symboliques, de "traces mythiques" aux "traces romanesques $» .^{13}$ La part subjective est évidemment privilégiée, pour filtrer ces traces et leur impact sur les personnages, et présentée de manière elliptique à travers une écriture fragmentée. Cette jeune femme appelée Yemna, qui est enfermée dans son mutisme, son délire, sa chambre, sa mémoire, rappelle à bien des égards d'autres personnages farèsiens, dont Jidda (Le Champ des oliviers, Mémoire de l'absent), qui s'exprime par son délire et ses dessins. Ainsi, mutité et images dessinées sont liées comme un mode d'expression graphique non pas compensatoire mais amené par le silence. Si une approche intertextuelle permet plus facilement au lecteur familier de l'univers poétique de Farès de comprendre la fonction des dessins de Khélif par rapport au mutisme de Yemna, il faudra certainement plus de 
temps aux autres lecteurs pour associer ces dessins à la vision subjective et intérieure de la jeune femme. Le lecteur pourra ainsi expérimenter la perte des repères réalistes à partir des premières images, se faisant le miroir d'une langue farèsienne en crise, en délire, que les illustrations de Khélif traduisent. Tout cet espace sans mots est ainsi nécessaire pour évoquer une mémoire de la trace et du palimpseste. Le texte de Farès ne nécessite pas une place prépondérante, comme le montrent ses poèmes, et sait s'épurer pour faire résonner la polysémie des mots, rendre visibles les silences et la déflagration du langage comme de l'histoire. Le résumé de Khélif clarifie l'histoire du livre mais il est évident que le lecteur doit reconstruire, par ces bribes de texte, des faits, des situations, des émotions, des souvenirs qui sont amenés progressivement comme un palimpseste mémoriel de couches de souvenirs fragmentaires et superposés. Dans ce contexte, les dessins constituent la majeure partie de l'espace de lecture mais les mots travaillent l'imaginaire du lecteur, faisant appel notamment à la polysémie. Pourtant, à la différence des autres œuvres, il faudra attendre cinq pages (ou dix vignettes) de dessins pour qu'apparaisse le texte de Farès, le lecteur ayant comme seul indice le titre du livre. Au fur et à mesure de ces 5 premières pages composées de nuages de cendre, laissant deviner une ville puis des ponts et l'Ile Saint-Louis, émerge un personnage, silhouette humaine faite de taches noires ou blanches selon les vignettes pour se détacher d'un fond opaque, qui se rapproche progressivement en plan rapproché sans que le lecteur ne puisse être assuré qu'il prendra en charge la narration. Cette expérience visuelle ne peut se résumer avec des mots ni avec la description proposée par l'éditeur, qui rappelle de manière allégorique la censure:

Un homme marche lentement, un livre à la main, le long des quais de la Seine. Ses pensées le portent loin, dans les montagnes du Titteri, à l'école française de la zaouia de Madala, enveloppé d'une dense fumée qui étouffe tout : le paysage, les hommes, les animaux. Dans l'école, que le feu consomme lentement, rôdent les hommes qui ont "détourné les fleuves du Livre vers les sources de l'Enfer". Sous le soleil masqué par un dense écran noir, ils effacent - au tranchant d'une lame de couteau - la vie, la parole, la mémoire. ${ }^{14}$

Cet homme ressemble à s'y méprendre à Nabile Farès (les quais de la Seine étant d'ailleurs des lieux familiers de l'écrivain), et le livre porte comme titre Un Passager de l'occident. ${ }^{15}$ Une certaine mise en abîme mémorielle, comme l'indique l'éditeur, offrira certainement une des clefs de lecture. Il s'agit non seulement de la mémoire de cet homme qui encercle le récit (puisqu'il est présent au début et à la fin du livre), mais également de celle de Yemna, qui se remémore la mémoire d'autres femmes du Titteri. Ce processus de mise en abîme est typique de l'écriture farèsienne. Il en est de même de la narration, éclatée entre plusieurs instances narratrices. Ce «Il» peut être appréhendé comme un masque autobiographique de Farès comme nous l'avions proposé dans " Nabile Farès au miroir de l'absent », les dessins de Khélif s'en faisant le miroir de même qu'ils permettent, par leur opacité, d'être le réceptacle de la mémoire des autres personnages et du lecteur, comme fonctionnent les taches d'encre utilisées par les psychologues pour faire parler les souvenirs refoulés. ${ }^{16}$ Selon cette logique, le personnage de Yemna peut s'interpréter comme un prolongement de la mémoire subjective farèsienne qui prend en charge l'autre au féminin, au masculin, au singulier, au pluriel. Les dessins représentent sa mémoire et ses émotions refoulées par le mutisme et la folie. Cette démultiplication des masques narratifs permet finalement d'inscrire l'être en devenir, en exil, en transit :

J'affirmais ceci. Qu'au lieu d'entretenir une forme historique et psychologique du moi. Je devais activer la forme latérale d'un moi qui ainsi, n'était plus l'origine de 
quelque chose, mais le moment de parcours d'un plus vaste ensemble que lui. C'est ainsi que je me suis mis à écrire. ${ }^{17}$

Ce vaste ensemble se tisse par échos, par liens entre les œuvres, entre les personnages, sous la forme d'un palimpseste de mots grâce à cette démultiplication narrative qui permet de replacer l'histoire subjective de Yemna dans une œuvre plus large dont le but est décrit par la fin de La Jeune Femme et la mort :

un au-delà de la tuerie, de cette tuerie vue, revenue, après un siècle, dans ces montagnes du Titteri. Comme si la terre, l'histoire locale des années 1840, plus tardivement 1870 en un autre siècle, quelque cent cinquante années après, s'étaient souvenues telle une douleur toujours vivante. Dont voici l'histoire.

Qu'en est-il des dessins? S'ils permettent de se faire le miroir de l'autre narratif et démultiplié, retrouve-t-on des traces d'un palimpseste?

Alors que dominent des planches constituées de manière similaire avec plusieurs vignettes sur chaque page, seule une double page contient l'illustration d'une ancienne carte du Nord de l'Algérie. Elle évoque un passé ancien et des traces mythiques telles que les « livres, chants, épopées, danses, tambours, médecines, masques » apportés par les caravanes depuis le XVIIIème siècle lors de leur remontée du Sud au Nord. Au bas de la page est dessinée une frise fleurie avec deux tombes près desquelles sortent de terre deux vases peints fissurés. De cette même frise part un arbre dont les feuilles s'épanouissent sur la Kabylie et sont figurées par un troupeau de chevaux. Au pied de cet arbre se trouvent un âne et une autre poterie fissurée. Le texte quant à lui évoque les «grandes manœuvres du Sud» et des nombreuses tribus de ce territoire avec les "légendes» et les "réminiscences d'une autre guerre». Sur la carte est précisé notamment par qui et quand ces terres ont été parcourues. Ainsi, cette double page illustre bien la superposition temporelle des migrations ainsi que les différentes représentations de l'espace, qu'il s'agisse d'une représentation cartographique ou symbolique, archéologique ou historique. Ce palimpseste de réminiscences comporte des zones effacées, illisibles, témoignant du passage du temps et de la censure graphique. A la différence des autres vignettes du livre dans lesquelles domine le contraste entre le noir et le blanc, dans cette double page tout est gris. Cette double page constitue certainement un véritable palimpseste mémoriel historique et mythique collectif, qui contraste avec les autres pages plus intimes des mémoires individuelles. Nous pouvons conclure que les dessins de Khélif offrent ainsi des visions diversifiées propres à cette mise en abîme narrative et mémorielle farèsienne, avec des trajectoires temporelles déflagrées qui hantent et construisent toute son œuvre afin de proposer un au-delà et un en deçà de la colonisation, de l'Histoire. Il y a donc toujours superposition des traces historiques (les guerres entre tribus) et des traces mythiques (apportées par l'oralité, les rituels évoqués par les motifs des poteries, la symbolique du bestiaire...), caractéristique que nous retrouvons dans cette double-page. Il reste néanmoins à démêler les traces romanesques entremêlées à ces autres traces historiques et mythiques. Les traces romanesques sont représentées graphiquement de manière homogène, offrant une même lumière noire. Composés d'un mélange de taches d'encre, de peinture à l'huile, de fusain, ces blocs sombres d'ombres sont également travaillés par des traits fins, des hachures. Les taches peuvent s'interpréter comme des espaces indélébiles, même si elles sont parfois délavées, floutées, estompées; mais restent toujours sombres. Les traits fins, quant à eux, sont des déchirures, des cicatrices. Il s'agit donc d'un graphisme très particulier qui place la matière plastique au cœur de la démarche de l'artiste, de même que Farès rappelait au lecteur l'importance de la 
typographie. Le lecteur se trouve ainsi devant des images qui peuvent lui dévoiler parfois des traces de silhouettes, de visages, des ombres, mais surtout des espaces de lumière, des éclaircies de voyance malgré l'aveuglement dont le thème est présenté dès les premiers mots en décrivant la fumée qui « obscurcit les yeux ». Certes, le texte de Farès aide à la compréhension des dessins mais ceux-ci n'ont pas pour fonction d'illustrer le texte, mais plutôt de présenter l'espace domestique et intérieur de Yemna, que ce soit sa chambre ou ses souvenirs. Les images suggèrent souvent la présence de la jeune femme, assise sur une chaise, regardant par une fenêtre figurée par un espace blanc rectangulaire. Mais Yemna ne constitue qu'une petite partie de l'image, le reste de l'espace étant envahi de taches diluées, de blocs sombres, de hachures. Domine une représentation abstraite de l'espace intérieur constitué de coulées, de traits qui évoquent le geste du peintre et les différentes trajectoires du stylo, du pinceau, du doigt, de l'eau. Les dessins de Khélif peuvent effectivement aveugler le lecteur, qui doit se concentrer pour voir émerger un sens de cette lumière obscure comme un palimpseste offert à ses yeux. Ils expriment cette désorientation spatiale, fragmentée, mélange d'espaces intérieurs, de larmes, de formes étranges dans lesquels peuvent se deviner des motifs. C'est la même démarche que Farès décrivait quand Abdenouar devait deviner « à travers ombres et langages, les contours et visibilités du dessin de Jidda. Prendre l'ombre et lui faire dire son intention de lumière [...] au lieu de l'impossible vide de sa mort, les lignes viennent, les assemblages, et les tensions du monde ${ }^{18}{ }^{18}$ Comme l'avoue Khélif, son travail consistait à représenter la déflagration subjective et historique évoquée par la poétique farèsienne pour «traduire le chaos psychique qui peut habiter ces personnages et puis aussi le terrible désordre de l'histoire qui se vit actuellement en Algérie $" .^{19}$ Cette traduction visuelle repose essentiellement sur une vision intériorisée, rendue possible par une approche plasticienne qui propose «une autre lecture et une autre écriture du monde pour explorer d'autres territoires, l'âme, l'obscur ». La tension entre figuration et abstraction est donc vitale pour se libérer du trait, de la ligne droite, du contour, pour que la peinture se fasse " par les taches, comme celles que l'on voit quand on ferme les yeux ». Quant à son utilisation restreinte des couleurs au profit de toutes les nuances du noir, du blanc et du gris, elle lui permet de chercher à imiter la réalité et non à la recopier. Et cette imitation est d'autant plus convaincante quand elle repose sur l'utilisation du fusain. Créé à partir de la carbonisation des arbres, il évoque doublement « un nuage de cendres » comme l'indique le texte de Farès, à partir de sa matière et de sa couleur et aussi « le ciel devenu tableau à cause de la fumée ». Ce sera la même chose avec l'encre diluée, utilisée pour suggérer « la pluie qui tombe ». Khélif étire ses dessins vers un ailleurs, « un autre lieu, une autre histoire, un autre temps que celui où vit depuis trente-deux ans déjà cette femme ». Se superpose ainsi à la présence de Yemna un paysage de lumière noire et de deuil. Les planches finales effacent définitivement Yemna pour ne laisser d'espace qu'à ce paysage de l'au-delà et de l'en deçà. Ce sera ensuite le tour du personnage qui ressemble à Nabile Farès, présent en plan rapproché, mais qui s'efface au fur et à mesure des vignettes en plan large, pour ne plus laisser voir que l'espace urbain, palimpseste superposant chaotiquement les traces de ponts, de bâtiments, de rues, avec en haut de l'image ce même nuage de cendres et de pluies. Finalement, la fumée des dessins est celle qui fait couler l'encre de l'écrivain, de même que la pluie des larmes refoulées, afin d'essayer de comprendre le sens de ces meurtres, de ces trajectoires. 
13 De même que les mots deviennent des sépultures pour ceux qui n'en ont pas, ces dessins deviennent les cendres de ces corps disparus, effacés, biffés, rayés du monde. Mais quelle est la fonction de ces absents entre encre et ancrage sinon d'offrir des traces pour un dialogue phénoménologique et éthique? Khélif et Farès veulent tous deux offrir un espace incantatoire contre le mutisme et le néant au risque d'une certaine opacité. De la même manière, Yemna attend «les rythmes des ensorceleurs, guérisseurs du désert [...] cet exorcisme désiré qui la libère pour un temps. Un temps court. Du mutisme, de l'effroi ... Elle appelle l'enfance égarée dans l'autre monde des ombres ». Cet autre monde illustré graphiquement par Khélif correspond à la définition de l'art moderne proposée notamment par Paul Klee dans Théorie de l'art moderne. ${ }^{20}$ L'art doit rendre visible une vision secrète et se place dans la rencontre fécondante du visible et de l'invisible. En créant du rythme par les formes par exemple, en faisant résonner la clarté avec l'obscurité, l'art moderne est plus intuitif et intérieur mais comment propose-t-il des images cathartiques et quelle est la place du lecteur?

\section{Un dialogue phénoménologique et éthique au-delà des censures}

Certaines images dans La Jeune Femme et la mort ont clairement une fonction protectrice. Tracées à la peinture blanche sur ces images de lumière noire, elles font émerger, chez Khélif, un bestiaire inexpliqué par le texte farèsien (oiseaux, chevaux) sinon comme "l'enfance égarée dans l'autre monde des ombres». Ces figures tracées par des traits blancs ou noirs fins peuvent être appréhendées comme des remparts contre les cauchemars et l'oubli. Le motif des oiseaux se superpose ainsi sur le visage endormi de Yemna cauchemardant, ou encore sur le couple d'instituteurs disparus. L'image d'un troupeau de cheval se trouve sur la couverture qui protège Yemna après son évanouissement suite au meurtre et à la séparation d'avec sa fille. Ce motif se répète également dans la double page du palimpseste recouvrant la carte de la Kabylie, évoquant autant une identité qu'un nomadisme à la symbolique mythique. Ainsi cette rencontre entre le visible et l'invisible, l'exil et le lieu, l'au-delà et l'en deçà caractérise une approche artistique qui démultiplie les possibilités polyphoniques et polygraphiques afin de permettre une certaine voyance qui appelle à la participation du lecteur. De même que Jidda initie à " un langage qui crée à mesure qu'il voit », le lecteur doit créer au fur et à mesure des pages un univers propre à son interprétation pour entrer ainsi dans l'immanence de l'immatériel, approche phénoménologique qui laisse place à l'expérience perceptive sensorielle, mémorielle et active du lecteur. ${ }^{21}$ Face à ces différentes images plus ou moins abstraites, ces vignettes plus ou moins homogènes, il sera libre de construire sa propre interprétation et faire dialoguer mot et image en privilégiant certaines vignettes. Nous proposons de nous arrêter sur la seule vignette verticale de tout le livre, marquant ainsi une rupture essentielle qui, selon nous, invite à une lecture phénoménologique. Elle représente une silhouette féminine, de blanc vêtue, occupant un quart de l'image et d'où émane une lumière qui s'élève en contraste avec un noir sombre qui constitue le reste de la vignette. Le texte nous suggère qu'il s'agit de Yemna qui voit au-delà des cendres l'origine même de ces cendres, l'origine même des incendies du monde. "Ce qu'elle regarde maintenant monter devant elle est cette courbe de feu brouillant le ciel selon les mouvements d'une torche toujours présente, devenue éternelle ». Devenue torche éternelle, Yemna s'est 
transformée en réceptacle de l'au-delà. Cette vignette permet ainsi un mouvement d'élévation comme le faisait le poème calligraphique vertical dans Le Champ des oliviers évoquant les lignes du livre devenu « douce marque », pour que :

demeure
non pas
l'homme
mais
l'enfant.
Non pas
les mots
mais
la forme.
Non pas
le sens
mais
l'enfance. ${ }^{22}$

Par sa verticalité, ce poème incantatoire aux nombreux échos permet de s'élever pour un court instant et sortir de la linéarité du texte farèsien qui semble pour le regard constituer un bloc sans échappatoire. De même, tout le reste de La Jeune Femme et la mort est constitué d'un espace horizontal qui n'offre pas d'issue. Cette image verticale sera d'ailleurs suivie par des vignettes sombres illustrant les puits de pétrole que cette lumière avait pour un temps sublimés. Cette référence au pétrole qui a transformé le paysage de l'ancien monde avec son poids économique, politique, écologique ne doit pas empêcher de lire dans cette image un autre monde, "comme si les flammes élancées vers le ciel depuis les puits devaient signifier autre chose que cette continuelle combustion de feu dans l'aridité de l'air ». Chercher un autre sens aux mots a toujours été le but de Farès : " il ne suffit pas de connaître le mot de chaque chose mais tous les mots de toutes les choses $» .{ }^{23}$ Parallèlement, la collaboration entre Farès et Khélif permet d'offrir un autre sens aux images. Il s'agit finalement de proposer un autre espace, cet « entredeux » de l'exil, de l'émigration, leitmotiv des études postcoloniales. Pour Khélif, cet ailleurs est un espace intérieur conséquent de l'exil. Né à Alger, en 1959, il arrive avec sa famille à Marseille à l'âge de 5 ans, ville qu'il n'a pas quittée, mais il confesse habiter symboliquement un autre espace, une autre langue de signes grâce au dessin :

À travers lui j'ai trouvé un lieu, celui de la feuille, celui de l'art, un lieu peut-être que je n'ai pas trouvé en France. Un autre lieu que celui que nous avons quitté làbas en Algérie, un lieu où je peux rencontrer l'autre, et où l'autre peut me rencontrer. ${ }^{24}$

Si les études postcoloniales ont bien montré les enjeux à l'œuvre pour ces écrivains et artistes de l'entre-deux tant au niveau linguistique, thématique qu'esthétique avec l'impossibilité d'une pure imitation pour une transformation d'un élément culturel en expression inédite, nous proposons dans cet article de n'en conserver qu'une expérience phénoménologique proposée au lecteur qui motive la création d'un troisième espace, conséquence d'un déplacement: cet autre espace qui permet la rencontre avec le censuré, le refoulé.

Préférant dans ses textes une description plus poétique que psychanalytique, et connaissant l'importance de la pensée d'Emmanuel Levinas pour Farès, nous proposons de privilégier une interprétation phénoménologique. «Il faut maintenant apprendre à fermer les yeux du corps ; ouvrir l'autre regard » dira-t-il en parlant d'Abdenouar, sorti de son mutisme grâce à ses dessins « dont chaque trait est vivant » et qui permettent la 
rencontre d'un autre qui appartient autant au domaine du refoulé qu'à un regard intérieur qui démultiplie la réalité : « je ne dis pas images car les lignes du dessin sont plus fortes que les paysages d'une image, et le dessin vient de moi, tandis que l'image viendrait d'ailleurs, comme dans un rêve ${ }^{25}$ Cette conception du dessin selon Farès invite ainsi à une interprétation moins psychanalytique que phénoménologique. Cette langue de l'autre ne doit pas être diagnostiquée, analysée, mais son délire opaque doit être apprécié dans sa fonction vitale de survie, sa fonction créatrice. De nouveau, la description du personnage de Jidda et de ses dessins nous permet de proposer une lecture dans ce sens. Ses dessins, dont elle seule parvient à transcrire les secrets, sont une nécessité de vie pour elle mais également pour Abdenouar :

Ces dessins qu'elle traçait et épinglait sur tous les murs de la chambre. Qu'il aimait regarder. A cause de toutes ces distorsions qui composaient les paysages et personnes [...] Sortes d'êtres placés ainsi dans la folie de son monde comme des épouvantails au milieu d'un champ. ${ }^{26}$

Ces dessins offrent un espace schizoïde plutôt qu'un espace schizophrénique. Pour définir cette conception originale de la spatialité inspirée de la phénoménologie, nous nous reportons à l'analyse faite par Jean Baptiste Faure dans « Schizo-analyse et espace schizoïde : Deleuze et Guattari face à l'espace phénoménologique ». ${ }^{27}$ Faire une " schizoanalyse » de la relation entre l'individu et son rapport à l'espace revient à recourir à la perception phénoménologique. Selon Faure, la schizo-analyse appréhende sans chercher à interpréter, comme le ferait la psychanalyse, les flux de désirs qui animent le sujet et ses productions. L'espace n'est plus à concevoir comme un espace mathématique, géométrique. Il est vécu subjectivement dans une discontinuité de l'espace: "Plus qu'à un espace conçu comme juxtaposition de points, le philosophe nous amène à penser l'espace comme superposition d'impressions ou d'intensités ». Cette conception semble convenir autant à la description des dessins de Jidda qu'à ceux de Khélif (représentant les paysages intérieurs de Yemna et par mise en abîme de Farès-Khélif) : «le personnage n'apparaît que sur fond de paysage, avec le paysage. Le personnage est donc inséparable de son paysage ", selon Faure. Il faut différencier la schizophrénie quand le sujet est aliéné et la schizoïdie quand il transforme son énergie en force créatrice, comme l'art abstrait qui n'a pas besoin de ressasser du même, du figuratif. Il n'est donc pas nécessaire de schématiser le délire, de le rationaliser mais il est important de lui laisser un espace d'expression des forces vitales, même s'il apparait comme opaque. Les dessins de Khélif ont ainsi pour fonction d'illustrer l'espace schizoïde de Yemna comme un troisième espace discontinu, lieu de trajectoires au-delà des frontières (géographiques ou idéologiques). La pensée de Yemna se libère du moi sans bouger de sa chambre pour faire entrer l'autre de l'exil, seul espace possible audelà des censures :

[Elle...] franchit les mers, heurte les rivages, affronte les douanes et s'initie aux rigueurs des contrôles, à l'exil des lieux, aux troubles des mémoires, aux réminiscences solitaires du monde et de la nuit, aux transits des ombres attablées chaque jour comme de vrais êtres civils discutant du passé, de l'avenir, du présent, toujours à la recherche de leurs propres instants de croyance et d'asile.

19 L'espace schizoïde de Yemna se fait réceptacle des flux de désirs et des espoirs des autres exilés de ce monde, même s'ils ne témoignent que de traces, de trajectoires d'un même paysage de cendres. Les dessins de Khélif peuvent ainsi s'appréhender comme un espace dans lequel le lecteur peut devenir paysage, peut se faire chandelle pour éclairer ces taches d'ombres et de lumières et lire sous ce linceul de cendres la parole intérieure 
de cette jeune femme mais également de toutes les autres voix du mutisme et de l'aveuglement.

Si Farès et Khélif témoignent de cette autre langue en cherchant à la représenter avec leurs mots, leurs images, le support même de la bande-dessinée peut être perçu comme une autre langue (de même que les contes, le théâtre, les jeux). Ces supports artistiques peuvent aider les enfants d'immigrés qui ont eu l'expérience de l'entre-deux notamment, avec qui tous deux à leur manière ont travaillé (Farès et ses nombreuses interventions dans les écoles et Khélif comme animateur de quartier). Selon Farès :

[il est nécessaire de travailler...] à leur passage possible dans l'autre langue [...]. Les enfants sont de plain-pied avec ce que l'art moderne a inventé en littérature, et dans l'espace sonore, visuel, pictural : bouger la langue, écrire comme le faisait Apollinaire, peindre dans un espace, cet espace mythique intérieur tel qu'il s'est trouvé élaboré, mis en évidence par Klee ou Kandinsky ou Miro, voilà ce qui permet aux enfants étrangers à la langue d'entrer, un peu plus rassurés, dans les contraintes vitales, nécessaires, de la langue. ${ }^{28}$

Il s'agit ainsi d'un engagement éthique qui sous-entend « d'être responsable de la vie de l'autre humain, mon semblable et dissemblable à la fois ".29 Farès n'a eu de cesse de s'engager par la forme et le fond de ses textes mais aussi par ses collaborations artistiques ou associatives. Il témoigne dans un de ces derniers textes pour son blog datant du 9 Février 2015 de sa volonté « de transmettre, c'est à dire, à plusieurs voix, mémoires, histoires, éducations de soi et de l'autre, et, surtout, de créer, créer de la vie sociale qui ne vit que de créations, de répétitions, innovations et créations ${ }^{30}{ }^{30}$ Outre l'apprentissage des matières classiques, Farès revendique d'accorder une place majeure à la création dont "les dessins de paysages que l'on aimerait visiter, inventer, les paysages que l'on aimerait fuir, ceux qu'on a quittés, des foules de paysages », nous donnant ici une définition que nous proposons d'appliquer aux dessins de Khélif dans lesquels se superposent ceux de Farès, de Yemna, de tous les personnages de rencontres et de créations.

\section{Conclusion}

Alors que de nombreuses bandes dessinées affichent désormais un didactisme historique, tel que le reportage dessiné de Désirée et Alain Frappier, Dans l'ombre de Charonne (2012) pour évoquer la manifestation du 8 février 1962 (préfacée à juste titre par l'historien Benjamin Stora), c'est avant tout en tant qu'expression de dénonciation que les bédéistes maghrébins des deux côtés de la méditerranée renouvellent leur approche de l'identité et de l'histoire de l'Algérie depuis l'Indépendance grâce aux éditeurs alternatifs. ${ }^{31}$ Ils se situent hors-histoire officielle et privilégient la petite histoire sur la grande Histoire en associant intimement recherche picturale et dénonciation sociale. Ces récits évitent l'exotisme pour retourner dans les non-dits, les trous obscurs de l'Histoire en assumant un ton sombre, comme nous l'avons montré avec notre lecture de La Jeune Femme et la mort. La bande dessinée selon Thierry Groensteen "même lorsqu'elle paraît désancrée, [...] donne prise à une lecture politique; il s'y inscrit toujours, fût-ce implicitement ou à l'insu de l'auteur, une vision du monde » en incarnant la résistance, en témoignant postérieurement des horreurs commises. ${ }^{32}$ La concision textuelle associée à la fulgurance du dessin permet une compréhension plus immédiate. Le dialogue entre le texte et les images, entre un écrivain et un dessinateur nous semble illustrer de la plus belle manière possible ce 
vivre-ensemble martelé à tout va et qui devrait s'appeler un «créer ensemble » pour aller en deçà et au-delà de la violence des censures. Il ne faut certes pas avoir peur d'assumer un langage de l'étrangeté, comme l'ont fait Farès et Khélif, soutenus par des éditeurs indépendants et dont l'étude autant que la promotion dans les écoles, les associations, restent vitales. Et pourtant l'espace pour « une approche poétisée de la réalité [soutenue par des éditeurs aujourd'hui sous pression économique...] s'est extrêmement restreint. Un livre comme La Jeune Femme et la mort ne pourrait pratiquement pas émerger aujourd'hui ", constate Khélif. ${ }^{33}$ Espérons que persisteront les héritiers de Nabile Farès, de cet engagement poétique et éthique, et de tous ces « autres » qui restent indispensables à notre survie.

\section{NOTES}

1. L'Armée de libération nationale (A.L.N), bras armé du Front de libération nationale impose une vision idéologique manichéenne et figée idéalisant la violence et le sacrifice.

En référence à l'étude de l'historien Benjamin Stora (La gangrène et l'oubli, Paris, La Découverte, 1991) qui analyse la double occultation mémorielle de la guerre d'Algérie : une "guerre sans nom » du côté français, une guerre pour l'indépendance unifiée du côté algérien qui omet les fractions internes et le massacre des opposants.

2. Contre la version officielle prônant l'arabe comme langue officielle du savoir, du sacré et du pouvoir politique, Farès témoigne d'un plurilinguisme historique et contemporain en Algérie (du berbère à l'espagnol) et défend la valeur de toutes les expressions (artistiques, populaires, orales ou encore 'névrosées' selon le discours médical).

Nabile Farès, Le Champ des oliviers, Paris, Le Seuil, 1972, p. 42. Ce titre sera désormais abrégé sous la forme Le Champ.

3. Ali Chibani, « Nabile Farès, à la mémoire de l'Absent », septembre 2016. https://orientxxi.info/ fr/auteur/ali-chibani. Chibani publiera bientôt aux éditions Koukou (Alger), avec Beida Chiki, un ouvrage en hommage à la disparition de Nabile Farès. Cet article reprend les idées d'un chapitre qui sera présent dans ce livre.

4. Karine Chevalier, La Mémoire et l'Absent. Nabile Farès et Juan Rulfo de la Trace au Palimpseste, Paris, L'Harmattan, 2008.

5. Farès, Le Champ, p. 42.

6. Farès, Le Champ, p. 173.

7. Ces maisons d'éditions jeunes et associatives (Rackham implantée à Tarnac depuis 1989, Amok 1994-2001 à Montreuil) s'intéressent à renouveler la relation entre texte et image en dialogue avec les réalités socio-historiques contemporaines.

8. Farès Nabile et Kamel Khélif, Les Exilées, histoires, Amok, 2001.

9. Farès Nabile et Kamel Khélif, La Petite Arabe qui aimait la chaise de Van Gogh, Amok, 2002.

Les pages n'étant pas numérotées, nous ne pouvons donc donner d'indication sur les extraits du récit dans les bandes-dessinées.

10. Farès Nabile et Kamel Khélif, La Jeune Femme et la mort, Rackham, 2010.

11. Selon la page d'accueil du site Frémok, http://www.bibliomonde.com/editeur/ fremok-300.html. 
12. Alain Paire, « Kamel Khélif, entre Marseille et Alger, en compagnie du Dahlia noir », paru le 16 mai 2008. https://www.nouvelobs.com/rue89/rue89-rue89-culture/20080516.RUE4227/kamelkhelif-entre-marseille-et-alger-en-compagnie-du-dahlia-noir.html.

13. Farès Nabile, Mémoire de l'absent, Paris, Le Seuil, 1974.

Les évènements historiques sont l'objet d'une traduction mythique dans les contes par exemple et romanesque par la création de personnages rejouant les actions dans un contexte contemporain.

14. http://www.editions-rackham.com/produit/la-jeune-femme-et-la-mort/.

15. Voir Farès Nabile, Un passager de l'Occident, Paris, Le Seuil, 1971.

16. Karine Chevalier, « Nabile Farès au miroir de l'absent », Cahiers de l'AIEF, 2005, numéro 57, pp. 365-376.

17. Farès, Le Champ, p. 188.

18. Farès, Mémoire, p. 226.

19. Alain Paire, «Kamel Khélif, entre Marseille et Alger, en compagnie du Dahlia noir », paru le 16 mai 2008. https://www.nouvelobs.com/rue89/rue89-rue89-culture/20080516.RUE4227/kamelkhelif-entre-marseille-et-alger-en-compagnie-du-dahlia-noir.html

20. Paul Klee, Théorie de l'art moderne, Paris, Gallimard, 1998 (1969).

21. Farès, Mémoire, p. 67.

22. Farès, Le champ, p. 198.

23. Farès, Mémoire, p. 95-96.

24. Caroline Trouillet, "Une ville d'exil, à l'encre de Kamel Khélif », paru le 25 Octobre 2017. http://africultures.com/marseille-a-lencre-de-kamel-khelif/

25. Farès, Mémoire, p. 226.

Farès, Mémoire, p. 106.

Farès, Mémoire, p. 108.

26. Farès, Le champ, p. 151.

27. Jean-Baptiste Faure, «Schizo-analyse et espace schizoïde : Deleuze et Guattari face à l'espace phénoménologique », Philosophique [Online], 15 | 2012, http://journals.openedition.org/ philosophique/556 https://doi.org/10.4000/philosophique.556

28. Azami-Tawil Bouthaina, Marzouki Samir, Sebbar Leila, Farès Nabile. Table ronde des écrivains. In: Cahiers de l'Association internationale des études francaises, 2005, $\mathrm{n}^{\circ} 57 . \mathrm{pp}$. 425-442.www.persee.fr/doc/caief_0571-5865_2005_num_57_1_1590 et plus précisément pp. 431-442.

29. Thompson Roger Williams Peter, «Interview Avec Nabile Farès ». http:// crisolenguas.uprrp.edu/Articles/PeterThompson.pdf

30. Farès Nabile, «Créer, accueillir, éduquer, transmettre ", publié le 9 Février 2015 sur son blog : https://blogs.mediapart.fr/nabile-fares/blog/090215/creer-accueillir-eduquer-

transmettre.

31. Frappier Désirée et Alain, Dans l'ombre de Charonne, Paris, Mauconduit, 2012.

32. Groensteen Thierry, La Bande dessinée : mode d'emploi, Bruxelles, Les Impressions nouvelles, 2008.

33. Laude Florence, «Rencontre avec Kamel Khélif», paru le 1 avril 2012. http:// imagesentete.blogspot.com/2012/04/rencontre-avec-kamel-khelif.html 


\section{RÉSUMÉS}

Cet article analyse comment Nabile Farès s'oppose aux censures idéologiques, historiques, politiques, linguistiques, politiques, éditoriales en conséquence de la guerre d'Algérie. Avec une écriture volontairement opaque, aux mots réprimés, dans laquelle le symbolisme visuel domine, il cherche à illustrer tout en combattant les silences de la censure. Ses dernières bandes dessinées (en collaboration avec l'illustrateur Kamel Khélif), peuvent se lire comme un espace intermédial polyphonique/ polygraphique offrant un dialogue éthique et phénoménologique qui témoigne du silence des mots tout en révélant les échos des images.

This article shows how Nabile Farès fight ideological, historical, linguistic, political, editorial censorships following the Algerian war of Independence. With his auto-censured opaque writing style in which dominate visual and typographic symbolism echoing repressed words, he intends to illustrate and fight against these silencing censorships. His latest graphic novels, in collaboration with the illustrator Kamel Khélif, can be read as an ethical and phenomenological dialogue based on polygraphic/polyphonic voices which testify of the silencing but reveals the echoes.

\section{AUTEUR}

\section{KARINE CHEVALIER}

Karine Chevalier est maître de conférences à l'Université de Roehampton, à Londres. Elle enseigne le cinéma et la littérature française et francophone. Titulaire d'un doctorat en littérature comparée, elle a publié notamment deux monographies chez L'Harmattan (La mémoire et l'absent. Nabile Farès et Juan Rulfo, de la trace au palimpseste, 2008 ; La mémoire et le présent. Daniel Maximin et Salman Rushdie, du masque au chaos, 2010) et de nombreux articles sur le cinéma. Sa recherche se concentre actuellement sur la représentation de la violence contemporaine dans le cinéma français.

Karine Chevalier is Senior Lecturer in French and Francophone studies at Roehampton University, London (U.K). Her main research interests lies in the field of French cinema and Francophone Postcolonial Studies. She has published two monographs on memory from both imaginative and cultural perspectives (La mémoire et l'absent. Nabile Farès et Juan Rulfo, de la trace au palimpseste, 2008; La mémoire et le présent. Daniel Maximin et Salman Rushdie, du masque au chaos, 2010) as well as numerous articles on French and Francophone literature and cinema. The main focus of her research is on the representation of violence in contemporary French cinema. 